\title{
Local Existence Theorems and Blow-up of Solutions for Quasi-linear Hyperbolic Cauchy Problems in a Domain with Characteristic Boundary Generated by Initial Data
}

By

\author{
Reiko SAKAMOTO*
}

\section{Introduction}

Non-degenerate quasi-linear hyperbolic equations are fairly well studied locally or globally under various boundary conditions. In degenerate case, recently, Y. Ebihara ([1]) showed a local existence theorem on one-dimensional elastic problem:

$$
\left\{\begin{array}{l}
\partial_{t}^{2} u=\partial_{x}\left\{\left(\partial_{x} u\right)^{m}\right\} \quad \text { in }\{0<t<T, 0<x<1\}, \\
\left.\partial_{x} u=0 \quad \text { on }\{0<t<T, x=0\} \cup 0<t<T, x=1\right\}, \\
u=\phi(x), \partial_{t} u=\psi(x) \quad \text { on }\{t=0,0<x<1\}
\end{array}\right.
$$

under some assumptions on $\{\phi(x), \psi(x)\}$ in case of $m \geqq 5$, which was extended in case of $m \geqq 3$ by $Y$. Nonaka ([2]). On the other hand, concerning to linear problem, fully degenerate hyperbolic Cauchy problems are studied by the author ([3]). A "fully degenerate" operator in a domain means an operator degenerating only on the boundary which is a characteristic whose order of multiplicity is equal to the order of the operator.

The aim of this paper is to prove a local existence theorem for a quasi-linear hyperbolic Cauchy problem:

$$
(P)\left\{\begin{array}{l}
A\left(t, x ; \phi^{m-1} u ; \partial\right) u \\
\equiv \partial_{t}^{m} u+\sum_{\substack{j+|v| \leqq m \\
j \leqq m-1}} a_{j v}\left(t, x ; \phi^{m-1} u\right) \partial_{t}^{j} \partial_{x}^{v} u \\
=f\left(t, x ; \phi^{m-1} u\right) \quad \text { in }(0, T) \times \Omega, \\
\partial_{t}^{j} u=\phi_{j}(x)(j=0,1, \cdots, m-1) \quad \text { on }\{t=0\} \times \Omega,
\end{array}\right.
$$

Communicated by S. Matsuura, August 31, 1988. Revised January 26, 1989.

* Department of Mathematics, Nara Women's University, Nara 630, Japan. 
where we use simple notations:

$$
\begin{aligned}
& \partial^{k} u=\left\{\partial_{t}^{j} \partial_{x}^{v} u|j+| v \mid=k\right\}, \\
& \partial^{k} u=\left\{\partial_{t}^{j} \partial_{x}^{v} u|j+| v \mid \leqq k\right\}=\left(u, \partial u, \cdots, \partial^{k} u\right),
\end{aligned}
$$

when there are no confusions.

The difficulty comes out of non-linearity, because degeneracy of coefficients depends on unknown solutions. This paper maintains that there is a unique classical solution in a short time for Cauchy problems in $(0, T) \times \Omega$ if initial data are "degenerate-structure generating". Under these situations, the analysis becomes very easy because the boundary values of solutions are determined only by the boundary values of initial data. The existence theorem is proved by the standard iterative method, using the existence theorems for iterative linearized problems ([3]). This paper also gives some examples of equations whose solutions blow-up on the boundary.

Similar results for special systems of equations are known in the fields of fluid dynamics, based on the theories of symmetric hyperbolic systems of first order or second order $([4],[5],[6])$.

\section{$\S 1$. Limear Carchy Problems for Funly Degenerate Hyperbolic Equarions}

Let us consider a linear hyperbolic equation in $I \times \Omega$ :

$$
A u \equiv \sum_{j+|v| \leqq m} a_{j v}(t, x) \partial_{t}^{j} \partial_{x}^{v} u=f(t, x),
$$

where $a_{m 0}=1$ and $a_{j v} \in \mathscr{B}^{\infty}(I \times \Omega)$, where

$$
\begin{aligned}
& I=(0, T), \Omega=R_{+}^{n}=\left\{x=\left(x_{1}, x^{\prime}\right) \mid x_{1}>0, x^{\prime} \in R^{n-1}\right\}, \\
& \partial_{x}=\left(\partial_{1}, \cdots, \partial_{n}\right), \partial_{j}=\partial_{x_{j}}(j=1,2, \cdots, n) .
\end{aligned}
$$

Let us introduce a function $\rho\left(x_{1}\right) \in \mathscr{B}^{\infty}\left(\mathbb{R}_{+}\right)$satisfying

$$
\rho\left(x_{1}\right)= \begin{cases}x_{1} & \text { if } x_{1}<1 / 2, \\ 1 & \text { if } x_{1}>1,\end{cases}
$$

and $0<\rho\left(x_{1}\right)<1$ if $1 / 2<x_{1}<1$.

Let us say that $\mathbb{A}$ is a hyperbolic equation with degenerate order $\sigma(\geqq 1)$ in $I$ $\times \Omega$, which we denote $A \in R H^{\sigma}(I \times \Omega ; \delta)(\delta>0)$, if

i) $\mathrm{a}_{j v}(t, x)=\rho\left(x_{1}\right)^{-\sigma|v|} a_{j v}(t, x)$ is bounded in $I \times \Omega$,

ii) $A_{\hat{0}}^{\wedge}(t, x ; \tau, \zeta)=\sum_{j+|v|=m} a_{j v}^{\wedge}(t, x) \tau^{j} \zeta^{v}=\prod_{k=1}^{m}\left(\tau-\tau_{k}^{\wedge}(t, x ; \zeta)\right)$

is a regularly hyperbolic polynomial in $I \times \Omega$, that is, $\left\{\tau_{\hat{k}}(t, x ; \zeta)\right\}$ are real for $(t, x) \in I \times \Omega$ and $\zeta \in S^{n-1}$, and moreover 


$$
\inf _{\substack{(t, x) \in I \times \Omega \\ \zeta \in S^{n-1}, k \neq k^{\prime}}}\left|\tau_{k}^{\wedge}(t, x ; \zeta)-\tau_{k^{\prime}}^{\wedge}(t, x ; \zeta)\right| \geqq \delta .
$$

We denote $A \in R H^{\sigma}(I \times \partial \Omega ; \delta)$, if $A \in R H^{\sigma}(I \times \Sigma ; \delta)$, where $\Sigma=\Omega \cap\left\{0<x_{1}\right.$ $\left.{ }^{\exists} \varepsilon\right\}$. Moreover, we denote $A \in R H^{\sigma}(I \times \Omega ; \delta ; s, K)\left(s \geqq s_{0}, K>0\right)$ if $A \in R H^{\sigma}(I \times \Omega ; \delta)$ and $a_{j v}$ are represented as $a_{j v}=a_{j v}^{\prime}+a_{j v}^{\prime \prime}$ with the estimate

$$
\sum_{j, v}\left|a_{j v}^{\prime}\right|_{\mathscr{B}^{s}(I \times \Omega)}+\sum_{j, v}\left\|a_{j v}^{\prime \prime}\right\|_{s} \leqq K,
$$

where

$$
\|u\|_{s}=\sup _{t \in I}\|u(t)\|_{s}=\sup _{t \in I} \sum_{j=0}^{s}\left\|\partial_{t}^{j} u(t)\right\|_{H^{s-J}(\Omega)},
$$

and $s_{0}=\langle m \sigma\rangle+[n / 2]+1$, where $\langle k\rangle$ means the least integer not less than $k$. Let us denote

$$
\tilde{a}_{j v}(t, x)=\rho\left(x_{1}\right)^{-\langle\delta|v|\rangle} a_{j v}(t, x) .
$$

Then we have

$$
\left|\tilde{a}_{j v}\right|_{\mathscr{B}^{s-s_{0}(I \times \Omega)}} \leqq C\left|a_{j v}\right|_{\mathscr{B}^{s-[n / 2]-1}(I \times \Omega)} \leqq C^{\prime} K
$$

Our problem in this section is the Cauchy problem:

$$
(P)_{l i n}\left\{\begin{array}{l}
A(t, x ; \partial) u=f(t, x) \quad \text { in } I \times \Omega, \\
\partial_{t}^{j} u=\phi_{j}(x)(j=0,1, \cdots, m-1) \quad \text { on }\{t=0\} \times \Omega .
\end{array}\right.
$$

and basic energy estimates have been obtained in [3]:

Lemma 1.1. There exists a positive constant $C$ such that

$$
\begin{aligned}
& \sum_{j+|v| \leqq m-1}\left\|\partial_{t}^{j}\left(\rho^{\sigma} \partial_{x}\right)^{v} u(t)\right\|^{2} \\
& \quad \leqq C\left\{\sum_{j=0|v| \leqq m-1-j}^{m-1} \sum_{1}\left\|\left(\rho^{\sigma} \partial_{x}\right)^{v} \phi_{j}\right\|^{2}+\int_{0}^{t}\|f(t)\|^{2} d t\right\}
\end{aligned}
$$

for any $A \in R H^{\sigma}(I \times \Omega ; \delta ; s, K)$ and any $u \in H^{m}(I \times \Omega)$, where \|\|$=\|\|_{L^{2}(\Omega)}$.

Here we remark a variation of Sobolev's lemma, which is familiar in the context of non-linear calculations.

Lemma 1.2. There exists a positive constant $C$ such that

$$
\sum_{\left|\alpha_{1}\right|+\ldots+\left|\alpha_{\ell}\right| \leqq s}\left\|\partial^{\alpha_{1}} v_{1} \cdots \partial^{\alpha_{\ell}} v_{\ell}\right\| \leqq C \prod_{j=1}^{\ell}\left\|v_{j}\right\|_{s}
$$

for any $v_{j} \in H^{s}(\Omega)(j=1, \cdots, \ell)$, where $s \geqq[n / 2]+1$.

Let us denote 


$$
\begin{aligned}
& \|\| u(t)\left\|_{s}^{2}=\sum_{j+|v| \leqq s}\right\| \partial_{t}^{j} \partial_{x}^{v} u(t) \|^{2}, \\
& \|u(t)\|_{(m-1)}^{2}=\sum_{j+|v| \leqq m-1}\left\|\partial_{t}^{j}\left(\rho^{\sigma} \partial_{x}\right)^{v} u(t)\right\|^{2},
\end{aligned}
$$

and

$$
\|u(t)\|_{(s)}^{2}=\sum_{j+|v| \geqq s-m+1}\left\|\partial_{t}^{j} \partial_{x}^{v} u(t)\right\|_{(m-1)}^{2}(s \geqq m),
$$

then we have

Lemma 1.3 . There exists a positive constant $C$ such that

$$
\sum_{|\mu| \leqq s}\left\|\partial^{\mu} A u-A \partial^{\mu} u\right\| \leqq C\|u(t)\| \|_{(s+m-1)}
$$

for any $A \in R H^{\sigma}(I \times \Omega ; \delta ; s, K)$ and any $u \in H^{s+m}(I \times \Omega)\left(s \geqq s_{0}\right)$.

Proof. Let us assume that $|\mu| \leqq s$ and $j+|v| \leqq m$. Let us denote

$$
\begin{aligned}
& \left\|\partial^{\mu} a_{j v} \partial_{t}^{j} \partial_{x}^{v} u-a_{j v} \partial_{t}^{j} \partial_{x}^{v} \partial^{\mu} u\right\| \\
& \quad \leqq C \sum_{|\mu,|=1}^{|\mu|}\left\|\left(\partial^{\mu^{\prime}} a_{j v}\right)\left(\partial_{t}^{j} \partial_{x}^{v} \partial^{\mu-\mu^{\prime}} u\right)\right\|=C \sum_{|\mu,|=1}^{|\mu|} I_{\mu \prime},
\end{aligned}
$$

and

$$
\begin{aligned}
I_{\mu \prime} \leqq & \left\|\left(\partial^{\mu^{\prime}} a_{j v}^{\prime}\right)\left(\partial_{t}^{j} \partial_{x}^{v} \partial^{\mu-\mu^{\prime}} u\right)\right\| \\
& +\left\|\left(\partial^{\mu^{\prime}} a^{\prime \prime}{ }_{j v}\right)\left(\partial_{t}^{j} \partial_{x}^{v} \partial^{\mu-\mu^{\prime}} u\right)\right\|=I_{\mu^{\prime}}^{\prime}+I^{\prime \prime}{ }_{\mu \prime} \cdot
\end{aligned}
$$

For $\left|\mu^{\prime}\right| \leqq\langle\sigma|\nu|\rangle$, we have

$$
\begin{aligned}
I_{\mu} & \leqq C\left|a_{j v}\right|_{\mathscr{B}\langle\sigma|v|\rangle}\left\|\rho^{\langle\sigma|v|\rangle-\left|\mu^{\prime}\right|} \partial_{t}^{j} \partial_{x}^{v} \partial^{\mu-\mu^{\prime}} u\right\| \\
& \leqq C\left|a_{j v}\right|_{\mathscr{B}\langle m \sigma\rangle}\left\|\phi^{s} u\right\|_{(m-1)} \leqq C K\|u\|_{(s+m-1)} .
\end{aligned}
$$

For $\left|\mu^{\prime}\right| \geqq\langle\sigma|v|\rangle+1(\equiv b)$, we have

$$
\begin{aligned}
I^{\prime}{ }_{\mu \prime} & \leqq\left|a_{j v}^{\prime}\right|_{\mathscr{B}^{s}}\left\|\partial_{t}^{j} \partial_{x}^{v} \partial^{\mu-\mu^{\prime}} u\right\| \\
& \leqq\left|a_{j v}^{\prime}\right|_{\mathscr{B}^{s}}\left\|\partial_{t}^{j} u\right\|_{s-1} \leqq K\|u\|_{(s+m-1)}
\end{aligned}
$$

and we have

$$
\begin{aligned}
I^{\prime \prime}{ }^{\prime} & \leqq C\left\|\phi^{b} a_{j v}^{\prime \prime}\right\|_{s-b}\left\|\partial_{t}^{j} \partial_{x}^{v} u\right\|_{s-b} \\
& \leqq C\left\|a_{j v}^{\prime \prime}\right\|_{s}\left\|\partial_{t}^{j} u\right\|_{s-1} \leqq C K\|u\| \|_{(s+m-1)},
\end{aligned}
$$

owing to Lemma.1.2.

Here we have 
Proposition 1.4. There exists a positive number $C$ such that

$$
\begin{aligned}
& \|u(t)\|_{(s+m-1)}^{2} \\
& \quad \leqq C\left\{\|u(0)\|_{(s+m-1)}^{2}+\int_{0}^{t}\left\|A u\left(t^{\prime}\right)\right\|_{s}^{2} d t^{\prime}\right\} \quad(0<t<T)
\end{aligned}
$$

for any $A \in R H^{\sigma}(I \times \Omega ; \delta ; s, K)$ and any $u \in H^{s+m}(I \times \Omega) \quad\left(s \geqq s_{0}\right)$.

Proof. Since

$$
A \partial^{\mu} u=\partial^{\mu} A u-\left(\partial^{\mu} A u-A \partial^{\mu} u\right) \quad(|\mu| \leqq s),
$$

we have from Lemma.1.1 and Lemma.1.3

$$
\|u(t)\|_{(s+m-1)}^{2} \leqq C\|u(0)\|_{(s+m-1)}^{2}+C \int_{0}^{t}\left\{\|A u(t)\|_{s}^{2}+\|u(t)\|_{(s+m-1)}^{2}\right\} d t .
$$

Hence we have

$$
\partial_{t} U(t) \leqq C U(t)+C F(t)
$$

where

$$
U(t)=\int_{0}^{t}\|u(t)\|_{(s+m-1)}^{2} d t \text { and } F(t)=\|u(0)\|_{(s+m-1)}^{2}+\int_{0}^{t}\|A u(t)\|^{2} d t,
$$

therefore we have

$$
U(t) \leqq e^{C t} F(t)
$$

Hence we have

$$
\|u(t)\|_{(s+m-1)}^{2} \leqq C\left(1+e^{C t}\right) F(t)
$$

Now, let $h$ be a real number, then we have

$$
\rho^{-h} A\left(t, x ; \partial_{t}, \partial_{x}\right) u=A\left(t, x ; \partial_{t}, \partial_{1}+h \rho^{\prime} / \rho, \partial_{2}, \cdots, \partial_{n}\right)\left(\rho^{-h} u\right),
$$

and we have

Lemma 1.5. There exists $K^{\prime}>0$ such that

$$
A\left(t, x ; \partial_{t}, \partial_{1}+h \rho^{\prime} / \rho, \partial_{2}, \cdots, \partial_{n}\right) \in R H^{\sigma}\left(I \times \Omega ; \delta ; s, K^{\prime}\right),
$$

if $A(t, x ; \partial) \in R H^{\sigma}(I \times \Omega ; \delta ; s, K)$.

Here we have from Proposition.1.4 and Lemma.1.5

Proposition 1.4'. For any real number $h$ and any integer $s\left(\geqq s_{0}\right)$, there exists a positive number $C$ such that 


$$
\begin{aligned}
& \left\|\rho^{-h} u(t)\right\|_{(s+m-1)}^{2} \\
& \quad \leqq C\left\{\left\|\rho^{-h} u(0)\right\|_{(s+m-1)}^{2}+\int_{0}^{t}\left\|\rho^{-h} A u\left(t^{\prime}\right)\right\|_{s}^{2} d t^{\prime}\right\} \quad(0<t<T)
\end{aligned}
$$

for any $A \in R H^{\sigma}(I \times \Omega ; \delta ; s, K)$ and any $u \in H^{s+m, h}(I \times \Omega)$, where $u \in H^{s, h}$ means that

$$
\|u\|_{H^{s, h}}=\left\|\rho^{-h} u\right\|_{H^{s}}<+\infty
$$

Let us say that $(P)_{l i n}$ is solvable in $H^{\infty, h}$ if there exists a unique solution in $H^{\infty, h}(I \times \Omega)$ for every data $\left\{f \in H^{\infty, h}(I \times \Omega), \phi_{j} \in H^{\infty, h}(\Omega)\right\}$. Then $(P)_{l i n}$ is solvable in $H^{\infty, h}$ if $A \in R H^{\sigma}(I \times \Omega ; \delta)([3])$.

Now, denoting

$$
\dot{a}_{j v, p}\left(t, x^{\prime}\right)=\left(\partial_{1}^{p} a_{j v}\right)\left(t, 0, x^{\prime}\right)
$$

we have

$$
\begin{aligned}
& A(t, x ; \partial)-\partial_{t}^{m}=\sum_{\substack{j+|v| \leqq m \\
j \leqq m}} a_{j v}(t, x) \partial_{t}^{j} \partial_{x}^{v} \\
& \quad \sim \sum_{\substack{j+|v| \leqq m \\
j \leqq m}} \sum_{p=|v|}^{\infty}(p !)^{-1} x_{1}^{p} \dot{a}_{j v, p}\left(t, x^{\prime}\right) \partial_{t}^{j} \partial_{x}^{v} \\
& \sim \sum_{p=0}^{\infty}(p !)^{-1} x_{1}^{p}\left\{\sum_{\substack{j+|v| \leqq m \\
j \leqq m \\
\left|v^{\prime}\right| \leqq p}} p !\left\{\left(p+v_{1}\right) !\right\}^{-1} \dot{a}_{j v, p+v_{1}}\left(t, x^{\prime}\right) \partial_{t}^{j} \partial_{x}^{v^{\prime}} x_{1}^{v_{1}} \partial_{1}^{v_{1}}\right\} \\
& \sim \sum_{p=0}^{\infty}(p !)^{-1} x_{1}^{p} B_{p}\left(t, x^{\prime} ; \partial_{t}, \partial_{x^{\prime}} ; x_{1} \partial_{1}\right) .
\end{aligned}
$$

Here we remark that

$$
\begin{aligned}
& B_{0}\left(t, x^{\prime} ; \partial_{t}, \partial_{x^{\prime}} ; x_{1} \partial_{1}\right) \\
& \quad=\sum_{\substack{j+k \leqq m \\
j \leqq m}}(k !)^{-1} \dot{a}_{j k 0, k}\left(t, x^{\prime}\right) \partial_{t}^{j}\left(x_{1} \partial_{1}-(k-1)\right)\left(x_{1} \partial_{1}-(k-2)\right) \cdots x_{1} \partial_{1},
\end{aligned}
$$

which is independent of $\partial_{x^{\prime}}$ Let $u(t, x) \in \mathscr{B}^{\infty}(I \times \Omega)$ and let $\dot{u}_{j}\left(t, x^{\prime}\right)=\left(\partial_{1}^{j} u\right)$ $\left(t, 0, x^{\prime}\right)$, then we have

$$
\begin{gathered}
\left\{A-\partial_{t}^{m}\right\} u(t, x) \sim \sum_{p=0}^{\infty} \sum_{q=0}^{\infty}(p ! q !)^{-1} x_{1}^{p+q} B_{p}\left(t, x^{\prime} ; \partial_{t}, \partial_{x^{\prime}} ; q\right) \dot{u}_{q}\left(t, x^{\prime}\right) \\
\sim \sum_{r=0}^{\infty}(r !)^{-1} x_{1}^{r}\left\{\sum_{p=0}^{r}\left(\begin{array}{l}
r \\
p
\end{array}\right) B_{p}\left(t, x^{\prime} ; \partial_{t}, \partial_{x^{\prime}} ; r-p\right) \dot{u}_{r-p}\left(t, x^{\prime}\right)\right\} .
\end{gathered}
$$

Proposition 1.6. Let us assume that

$$
f(t, x) \in H^{\infty}(I \times \Omega), \phi_{j}(x) \in H^{\infty}(\Omega) .
$$


For any positive integer $h$, there exist

$$
\left\{g_{j}\left(t, x^{\prime}\right)\right\}_{0 \leqq j \leqq h-1} \subset H^{\infty}(I \times \partial \Omega)=H^{\infty}\left(I \times R^{n-1}\right),
$$

satisfying

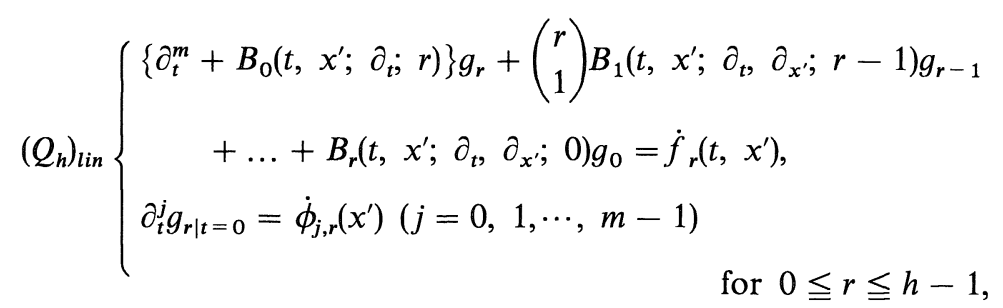

where

$$
\dot{f}_{p}=\left(\partial_{1}^{p} f\right)\left(t, 0, x^{\prime}\right), \quad \dot{\phi}_{j, p}=\left(\partial_{1}^{p} \phi_{j}\right)\left(t, 0, x^{\prime}\right) .
$$

We say that $g(t, x) \in \mathscr{B}^{\infty}(I \times \Omega)$ is an approximate function of order $h$ corresponding to the data $\left\{f(t, x) \in \mathscr{B}^{\infty}(I \times \Omega), \phi_{j}(x) \in \mathscr{B}^{\infty}(\Omega)\right\}$, if

$$
\left\{\begin{array}{l}
(A g)(t, x)-f(t, x) \in H^{\infty, h}(I \times \Omega), \\
\left(\partial_{t}^{j} g\right)(0, x)-\phi_{j}(x) \in H^{\infty, h}(\Omega)(j=0,1, \cdots, m-1) .
\end{array}\right.
$$

Let $\left\{g_{j}\right\}_{0 \leqq j \leqq h-1}$ satisfy $\left(Q_{h}\right)_{l i n}$, and set

$$
g(t, x)=\sum_{j=0}^{h-1}(j !)^{-1} \tilde{\rho}\left(x_{1}\right)^{j} g_{j}\left(t, x^{\prime}\right),
$$

where $\tilde{\rho}\left(x_{1}\right) \in C_{0}^{\infty}(R)$ and $\tilde{\rho}\left(x_{1}\right)=x_{1}$ near $x_{1}=0$. Then $g(t, x)$ is an approximate function of order $h$ corresponding to $\left\{f(t, x) \in H^{\infty}(I \times \Omega), \phi_{j}(x) \in H^{\infty}(\Omega)\right\}$. When $g(t, x)$ is an approximate function of order $h$ corresponding to $\{f(t$, $\left.x) \in H^{\infty}(I \times \Omega), \phi_{j}(x) \in H^{\infty}(\Omega)\right\}$, set

$$
\left\{\begin{array}{l}
f^{\prime}(t, x)=f(t, x)-(A g)(t, x) \in H^{\infty, h}(I \times \Omega), \\
\phi_{j}^{\prime}(x)=\phi_{j}(x)-\partial_{t}^{j} g(0, x) \in H^{\infty, h}(\Omega),
\end{array}\right.
$$

then $(P)_{l i n}$ is reduced to

$$
\begin{aligned}
& A\left(t, x ; \partial_{t}, \partial_{x}\right) u^{\prime}=f^{\prime}(t, x) \text { in } I \times \Omega, \\
& \left(P^{\prime}\right)_{\text {lin }} \\
& \partial_{t}^{j} u^{\prime}=\phi_{j}^{\prime}(x)(j=0,1, \cdots, m-1) \text { on }\{t=0\} \times \Omega \text {, }
\end{aligned}
$$

\section{$\S$ 2. Local Existence Theorems for Quasi-linear Cauchy Problems with Sufficiently Degenerate Data}

Let us consider a quasi-linear Cauchy problem: 
$(P)\left\{\begin{aligned} A\left(t, x ; \phi^{m-1} u ; \partial\right) u & \equiv \sum_{j+|v|=m} a_{j v}\left(t, x ; \phi^{m-1} u\right) \partial_{t}^{j} \partial_{x}^{v} u \\ & =f\left(t, x ; \phi^{m-1} u\right) \quad \text { in } I \times \Omega, \\ \partial_{t}^{j} u=\phi_{j}(x)(j=0,1, \ldots, m-1) & \text { on }\{t=0\} \times \Omega,\end{aligned}\right.$

where we assume $(A),(\sigma-S)$ and $(h-D)$ as follows. We denote

$$
\begin{aligned}
U_{k} & =\phi_{t}^{m-1-k} \partial_{x}^{k} u=\partial_{x}^{k}\left(u, \partial_{t} u, \cdots, \partial_{t}^{m-1-k} u\right), U=\left(U_{0}, U_{1}, \cdots, U_{m-1}\right), \\
\Phi_{k} & =\partial_{x}^{k}\left(\phi_{0}, \phi_{1}, \cdots, \phi_{m-1-k}\right), \quad \Phi=\left(\Phi_{0}, \Phi_{1}, \cdots, \Phi_{m-1}\right), \\
\text { and } N & =\sum_{k=0}^{m-1}(m-k)\left(\begin{array}{c}
k+n-1 \\
\mathrm{n}-1
\end{array}\right) .
\end{aligned}
$$

(A): i) $a_{j v}(t, x ; U) \in C^{\infty}\left(\overline{I \times \Omega} \times R^{N}\right)$,

ii) $A(0, x ; \Phi(x) ; \partial) \in R H^{0}\left(I \times \Omega_{\varepsilon}\right)$ for any $\varepsilon>0$, where $\Omega_{\varepsilon}=\Omega \cap\left\{x_{1}>\varepsilon\right\}$,

iii) $f(t, x ; U) \in C^{\infty}\left(\overline{I \times \Omega} \times R^{N}\right)$,

iv) $\phi_{j}(x) \in H^{\infty}(I \times \Omega)$.

$(\sigma-\mathbb{S}):$ i) $A(t, x ; 0 ; \partial) \in R H^{\sigma}(I \times \partial \Omega ; \delta)$,

ii) $f_{U_{k}}\left(t, x\right.$;0) $\rho\left(x_{1}\right)^{-k \sigma}$ is bounded in $I \times \Omega$.

$\left(R_{R}-\mathbb{D}\right)$ : i) $f(t, x ; 0) \in H^{\infty, h}(I \times \Omega)\left(h \geqq h_{0}\right)$,

ii) $\phi_{j}(x) \in H^{\infty, h}(\Omega)\left(h \geqq h_{0}\right)$, where $h_{0}=2\langle m \sigma\rangle$.

Theorem 1. Under the assumptions $(A),(\sigma-S)$ and $(h-D)$, there exists a unique solution of $(P)$ in $H^{s, h}\left(I_{0} \times \Omega\right)\left(s \geqq s_{0}\right)$, where $I_{0}=\left(0, T_{0}\right)$, if $T_{0}=T_{0}(s, h)(>0)$ is chosen small enough.

Taking $v=u-\sum_{j=0}^{m-1}(j !)^{-1} t^{j} \phi_{j}(x)$ for $u$, the Cauchy problem $(P)$ can be reduced to the Cauchy problem with zero initial data:

$$
(P-0)\left\{\begin{array}{l}
A\left(t, x ; \phi^{m-1} u, \partial\right) u=f\left(t, x ; \phi^{m-1} u\right) \text { in } I \times \Omega, \\
\not_{t}^{m-1} u=0 \text { on }\{t=0\} \times \Omega,
\end{array}\right.
$$

where $(A),(\sigma-S),(h-D)$ are satisfied with $\Phi=0$. Therefore, Theorem 1 will be shown for $(P-0)$ by using the method of successive approximation. Namely, we shall find $u^{(k)} \in H^{\infty, h}\left(I_{0} \times \Omega\right)$ satisfying

$$
\left(P^{(k)}-0\right)\left\{\begin{array}{l}
A\left(t, x ; \phi^{m-1} u^{(k-1)}, \partial\right) u^{(k)}=f\left(t, x ; \phi^{m-1} u^{(k-1)}\right) \quad \text { in } I \times \Omega, \\
\phi_{t}^{m-1} u^{(k)}=0 \text { on }\{t=0\} \times \Omega,
\end{array}\right.
$$

if $u^{(k-1)} \in H^{\infty}\left(I_{0} \times \Omega\right)$, and then we shall see that $u^{(k)} \rightarrow u$ in $H^{s-1, h}\left(I_{0} \times \Omega\right)$. 
Lemma 2.1. There exists $C>0$ such that

$$
\left\|a(x, u(x)) v_{1}(x) \cdots v_{\ell}(x)\right\|_{s} \leqq C|a|_{\mathscr{B}^{s}\left(\Omega \times B_{r}\right)}\left(1+\|u\|_{s}\right)^{s} \prod_{j=1}^{\ell}\left\|v_{j}\right\|_{s}
$$

for any $a(x, u) \in \mathscr{B}^{s}\left(\Omega \times R^{N}\right)$ and any $u(x), v(x) \in H^{s}(\Omega)$, satisfying $u \in B_{r}=\{u|| u(x) \mid$ $\leqq r\}(s \geqq[n / 2]+1, r>0)$.

Proof. We only see the case when $N=1$. Let $|v| \leqq s$. We remark that

$$
\begin{aligned}
\partial^{v}\{ & \left.a(x, u(x)) v_{1}(x) \cdots v_{\ell}(x)\right\} \\
= & \sum C_{\mu}\left\{\partial^{v-\mu_{1}-\cdots-\mu_{\ell}} \iota(x, u(x))\right\}\left(\partial^{\mu_{1}} v_{1}\right)(x) \cdots\left(\partial^{\mu} \ell v_{\ell}\right)(x) \\
= & \sum C_{\alpha k \beta}\left(\partial_{x}^{\alpha} \partial_{u}^{k} a\right)(x, u(x))\left(\partial^{\beta_{1}} u\right)(x) \ldots\left(\partial^{\beta_{k}} u\right)(x) \\
& \times\left(\partial^{\mu_{1}} v_{1}\right)(x) \ldots\left(\partial^{\mu} \ell v_{\ell}\right)(x),
\end{aligned}
$$

where $\sum$ is taken over

$$
|\alpha|+\left|\beta_{1}\right|+\cdots+\left|\beta_{k}\right|+\left|\mu_{1}\right|+\cdots+\left|\mu_{\ell}\right| \leqq|v|,
$$

where $\left|\beta_{j}\right| \geqq 1$. Owing to Lemma.1.2, we have

$$
\begin{aligned}
& \left\|\partial^{v}\left\{a(x, u(x)) v_{1}(x) \cdots v_{\ell}(x)\right\}\right\| \\
& \quad \leqq C|a|_{\mathscr{B}^{s}\left(\Omega \times B_{r}\right)} \sum_{k=0}^{s}\|u\|_{s}^{k} \prod_{j=1}^{\ell}\left\|v_{j}\right\|_{s} .
\end{aligned}
$$

Let us denote

$$
H_{\varepsilon(s, M)}^{\infty, h}(I \times \Omega)=\left\{u \in H^{\infty, h}(I \times \Omega) \mid\left\|\rho^{-h} u\right\|_{s_{0}} \leqq \varepsilon,\left\|\rho^{-h} u\right\|_{(s)} \leqq M\right\},
$$

then we have a fundamental lemma to get an iterative scheme.

Lemma 2.2. Let $s \geqq s_{0}$ and $h \geqq h_{0}$, then there exist positive constants $\varepsilon$ and $K_{0}$, independent of $K$, such that

i) $\quad A\left(t, x ; \not^{m-1} u ; \partial\right) \in R H^{\sigma}(I \times \Omega ; \delta / 2 ; s, 2 K)$,

ii) $\quad\left\|\rho^{-h} f\left(t, x ; \phi^{m-1} u_{s}\right)\right\| \leqq 2 K$,

if

i) $A(t, x ; 0 ; \partial) \in R H^{\sigma}(I \times \Omega ; \delta ; s, K)$,

ii) $\quad\left\|\rho^{-h} f(t, x ; 0)\right\| \|_{s} \leqq K$,

iii) $u \in H_{\varepsilon\left(s+m-1, M_{K}\right)}^{\infty, h}(I \times \Omega)$, where $M_{K}=\left(K / K_{0}\right)^{1 /(s+2)}-1$

for $K>K_{0}$.

Proof of i)'. Let us denote 


$$
\begin{aligned}
a_{j v}(t, x ; U) & =a_{j v}(t, x ; 0)+\sum_{k} \int_{0}^{1} a_{j v U_{k}}(t, x ; \theta U) d \theta U_{k} \\
& =a_{j v}(t, x ; 0)+\sum_{k} X_{j v k}(t, x ; U) U_{k},
\end{aligned}
$$

where $X_{j v k} \in C^{\infty}\left(\overline{I \times \Omega} \times R^{N}\right)$, that is, $\left|X_{j v k}\right|_{\mathscr{B}^{s}\left(I \times \Omega \times B_{r}\right)} \leqq C_{s, r}$.

We remark that

$$
\begin{aligned}
& \left|\rho^{-h+m-1} U\right|=\left|\rho^{-h+m-1} \not^{m-1}\left\{\rho^{h}\left(\rho^{-h} u\right)\right\}\right| \\
& \quad \leqq C\left|\phi^{m-1}\left(\rho^{-h} u\right)\right| \leqq C^{\prime}\left\|\rho^{-h} u\right\|_{m+[n / 2]} \leqq C^{\prime}\left\|\rho^{-h} u\right\|_{s_{0}},
\end{aligned}
$$

that is, we have

$$
|U(t, x)| \leqq C \rho^{h-(m-1)}\left\|\rho^{-h} u\right\|_{s_{0}} \leqq C \varepsilon \rho^{h-(m-1)} .
$$

Hence we have

$$
\left|a_{j v}(t, x ; U)-a_{j v}(t, x ; 0)\right| \leqq C \varepsilon \rho^{h-(m-1)} \leqq C \varepsilon \rho^{\sigma|v|} \quad\left(h \geqq h_{0}\right),
$$

because $h_{0} \geqq m-1+\sigma m$. Since $A(t, x ; 0 ; \partial) \in R H^{\sigma}(I \times \Omega ; \delta)$, we have $A(t, x ; U$; $\partial) \in R H^{\sigma}(I \times \Omega ; \delta / 2)$, taking $\varepsilon$ small enough. Moreover, we may assume that $|U(t, x)| \leqq 1$, taking $\varepsilon$ small enough.

Next, let $s \geqq s_{0}$ and $|v| \leqq s$. Owing to Lemma.2.1, we have

$$
\left\|X_{j v k}(t, x ; U) U_{k}\right\|_{s} \leqq C\left(1+\|u\|_{s+m-1}\right)^{s}\|u\|_{s+m-1} .
$$

We remark that

$$
\begin{aligned}
&\|u\|_{s+m-1}=\left\|\rho^{h}\left(\rho^{-h} u\right)\right\|_{s+m-1} \\
& \leqq C\left\{\left\|\rho^{h} \not^{s+m-1}\left(\rho^{-h} u\right)\right\|\right.+\left\|\rho^{h-1} \phi^{s+m-2}\left(\rho^{-h} u\right)\right\|+\ldots \\
&\left.+\left\|\rho^{h-(m-1)} \phi^{s}\left(\rho^{-h} u\right)\right\|+\left\|\rho^{-h} u\right\|_{s-1}\right\} \\
& \leqq C^{\prime}\left\|\rho^{-h} u\right\|_{(s+m-1)}\left(h \geqq h_{0}\right),
\end{aligned}
$$

because $h_{0} \geqq(m-1) \sigma$. Hence we have

$$
\begin{aligned}
& \sum_{j, v}\left\|a_{j v}(t, x ; U)-a_{j v}(t, x ; 0)\right\| \|_{s} \leqq C_{1}\left(1+\left\|\rho^{-h} u\right\|_{(s+m-1)}\right)^{s+1} \\
& \quad \leqq C_{1}\left(1+M_{K}\right)^{s+1} \leqq K
\end{aligned}
$$

for $K>K_{0}, K_{0} \geqq C_{1}$. Here we have

$$
a_{j v}(t, x ; U)=a_{j v}^{\prime}(t, x ; 0)+\left\{a_{j v}^{\prime \prime}(t, x ; 0)+\left[a_{j v}(t, x ; U)-a_{j v}(t, x ; 0)\right]\right\},
$$

where 


$$
\begin{aligned}
\sum_{j, v}\left|a_{j v}^{\prime}(t, x ; 0)\right|_{\mathscr{B}^{s}}+ & \sum_{j, v}\left\|\mid a_{j v}^{\prime \prime}(t, x ; 0)+\left[a_{j v}(t, x ; U)-a_{j v}(t, x ; 0)\right]\right\| \|_{s} \\
\leqq & \sum_{j, v}\left|a_{j v}^{\prime}(t, x ; 0)\right|_{\mathscr{B}^{s}}+\sum_{j, v} \mid\left\|a_{j v}^{\prime \prime}(t, x ; 0)\right\|_{s} \\
& \quad+\sum_{j, v}\left\|\mid a_{j v}(t, x ; U)-a_{j v}(t, x ; 0)\right\|_{s} \leqq 2 K .
\end{aligned}
$$

Hence we have $A(t, x ; U ; \partial) \in R H^{\sigma}(I \times \Omega ; \delta / 2 ; s, 2 K)$.

Proof of ii)'. In the same way as in the above, we have

$$
f(t, x ; U)=f(t, x ; 0)+\sum_{k=0}^{m-1} \xi_{k}(t, x ; U) U_{k}
$$

where $\xi_{k} \in C^{\infty}\left(\overline{I \times \Omega} \times R^{N}\right)$ and

$$
\left|\xi_{k}(t, x ; 0)\right| \leqq C \rho^{\langle k \sigma\rangle}
$$

Moreover, we have

$$
\xi_{k}(t, x ; U)=\xi_{k}(t, x ; 0)+\sum_{i} \eta_{k i}(t, x ; U) U_{i}
$$

where

$$
\rho^{-\langle k \sigma\rangle} \xi_{k}(t, x ; 0) \in \mathscr{B}^{\infty}(I \times \Omega), \quad \eta_{k i} \in C^{\infty}\left(\overline{I \times \Omega} \times R^{N}\right)
$$

Since

$$
\begin{aligned}
& \rho^{-h} \xi_{k}(t, x ; U) U_{k} \\
& =\left\{\rho^{-\langle k \sigma\rangle} \xi_{k}(t, x ; 0)\right\}\left\{\rho^{-h+\langle k \sigma\rangle} U_{k}\right\} \\
& \quad+\sum_{i} \eta_{k i}(t, x ; U)\left\{\rho^{-\langle k \sigma\rangle} U_{i}\right\}\left\{\rho^{-h+\langle k \sigma\rangle} U_{k}\right\},
\end{aligned}
$$

we have, owing to Lemma.2.1,

$$
\begin{aligned}
&\left\|\rho^{-h} \xi_{k}(t, x ; U) U_{k}\right\| \|_{s} \\
& \leqq C\left\{\left\|\rho^{-\langle k \sigma\rangle} \xi_{k}(t, x ; 0)\right\|_{s}\left\|\rho^{-h+\langle k \sigma\rangle} U_{k}\right\|_{s}\right. \\
&\left.+\left(1+\|U\| \|_{s}\right)^{s}\left\|\rho^{-\langle k \sigma\rangle} U\right\|\left\|_{s}\right\| \rho^{-h+\langle k \sigma\rangle} U_{k} \|_{s}\right\} \\
& \leqq C^{\prime}\left\{\left\|\rho^{-h+\langle k \sigma\rangle} U_{k}\right\|_{s}+\left(1+\|U\|\left\|_{s}^{s}\right\| \rho^{-h+\langle k \sigma\rangle} U_{k} \|_{s}^{2}\right\}\right.
\end{aligned}
$$

$\left(h \geqq h_{0}\right)$, because $h_{0} \geqq 2\langle(m-1) \sigma\rangle$. Hence we have

$$
\left\|\rho^{-h} \xi_{k}(t, x ; U) U_{k}\right\|_{s} \leqq C_{2}\left(1+\left\|\rho^{-h} u\right\|_{(s+m-1)}\right)^{s+2} \leqq C_{2}\left(1+M_{K}\right)^{s+2} \leqq K,
$$

therefore 


$$
\left\|\rho^{-h} f(t, x ; U)\right\|_{s} \leqq 2 K
$$

for $K>K_{0}$ if $K_{0} \geqq C_{2}$. Here we have only to set $K_{0}=\max \left(C_{1}, C_{2}\right)$.

Hereafter, we fix $K$ and $M=M_{K}$ so that $K$ satisfies (i), (ii) and $K>K_{0}$ in Lemma. 2.2.

Proposition 2.3. There exists $I_{1}=\left(0, T_{1}\right)\left(T_{1}>0\right)$ such that there exists a solution $\tilde{u} \in H_{\varepsilon(s+m-1, M)}^{\infty, h}\left(I_{1} \times \Omega\right)$ of the linearized problem:

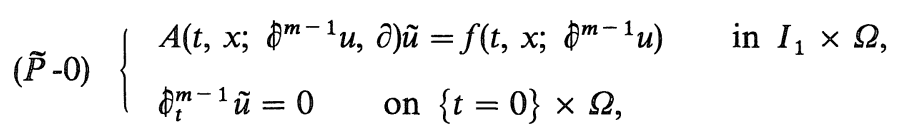

if $u \in H_{\varepsilon(s+m-1, M)}^{\infty, h}\left(I_{1} \times \Omega\right)$.

Proof. Applying Lemma.2.2, we have

$$
A\left(t, x ; \not^{n-1} u, \partial\right) \in R H^{\sigma}(I \times \Omega ; \delta / 2 ; s, 2 K) .
$$

Hence, applying Proposition.1.4', we can find a unique solution $\tilde{u}$ of $(\widetilde{P}-0)$ for any $u \in H_{\varepsilon(s+m-1, M)}^{\infty, h}(I \times \Omega)$, and

$$
\begin{aligned}
& \|\| \rho^{-h} \tilde{u}(t) \|_{(s+m-1)}^{2} \\
& \quad \leqq C \int_{0}^{t}\left\|\rho^{-h} f\left(t, x ; \phi^{m-1} u\right)\right\|_{s}^{2} d t \leqq C_{1} t .
\end{aligned}
$$

Taking

$$
T_{1}=\min \left(\varepsilon^{2}, M^{2}\right) / C_{1},
$$

we have $\tilde{u} \in H_{\varepsilon(s+m-1, M)}^{\infty, h}(I \times \Omega)$.

Following to Proposition.2.3, $u_{k} \in H_{\varepsilon(s+m-1, M)}^{\infty, h}\left(I_{1} \times \Omega\right)$ can be defined as a solution of $\left(P^{(k)}-0\right)$ successively, taking $u_{0}=0$. Let us denote

$$
\begin{aligned}
A(t, & \left.x ; \phi^{m-1} u^{(k-1)} ; \partial\right)\left(u^{(k)}-u^{(k-1)}\right) \\
= & -\left\{A\left(t, x ; \phi^{m-1} u^{(k-1)} ; \partial\right)-A\left(t, x ; \phi^{m-1} u^{(k-2)} ; \partial\right)\right\} u^{(k-1)} \\
& +\left\{f\left(t, x ; \phi^{m-1} u^{(k-1)}\right)-f\left(t, x ; \phi^{m-1} u^{(k-2)}\right)\right\} \\
= & g_{k}^{\prime}+g_{k}^{\prime \prime}=g_{k}(k=2,3, \cdots) .
\end{aligned}
$$

Then we have

Lemma 2.4.

$$
\left\|\rho^{-h} g_{k}\right\|_{s-1} \leqq C\left\|\rho^{-h}\left(u^{(k-1)}-u^{(k-2)}\right)\right\|_{(s+m-2)}(k=2,3, \cdots),
$$

Proof. Using Lemma.2.1, we have 


$$
\begin{aligned}
& \left\|\rho^{-h} g_{k}^{\prime}\right\|_{s-1} \leqq C\left(1+\left\|u^{(k-1)}\right\|_{s+m-2}+\left\|u^{(k-2)}\right\|_{s+m-2}\right)^{s-1} \\
& \times\left\|\rho^{-\langle(m-1) \sigma\rangle}\left(u^{(k-1)}-u^{(k-2)}\right)\right\|_{s+m-2}\left\|\rho^{-h+\langle(m-1) \sigma\rangle} \phi^{m} u^{(k-1)}\right\|_{s-1} \\
& \leqq \\
& \quad C^{\prime}\left(1+\left\|\rho^{-h} u^{(k-1)}\right\|_{(s+m-1)}+\left\|\rho^{-h} u^{(k-2)}\right\|_{(s+m-1)}\right)^{s} \\
& \quad \times\left\|\rho^{-h}\left(u^{(k-1)}-u^{(k-2)}\right)\right\|_{(s+m-2)},
\end{aligned}
$$

because $h_{0} \geqq 2\langle(m-1) \sigma\rangle$. In the same way, we have

$$
\begin{aligned}
& \left\|\rho^{-h} g_{k}^{\prime \prime}\right\| \|_{s-1} \\
& \leqq C^{\prime}\left(1+\left\|\rho^{-h} u^{(k-1)}\right\|_{(s+m-2)}+\left\|\rho^{-h} u^{(k-2)}\right\|_{(s+m-2)}\right)^{s-1} \\
& \quad \times\left\|\rho^{-h}\left(u^{(k-1)}-u^{(k-2)}\right)\right\|_{(s+m-2)} .
\end{aligned}
$$

Let us denote

$$
\mathscr{H}^{s, h}(I \times \Omega)=\left\{u||\left\|\rho^{-h} u\right\|_{(s)}<+\infty\right\}
$$

Then we have

Proposition 2.5. There exists $I^{\prime}=\left(0, T^{\prime}\right)\left(0<T^{\prime} \leqq T_{1}\right)$ such that

$$
u^{(k)} \longrightarrow u \quad \text { in } \mathscr{H}^{s+m-2, h}\left(I^{\prime} \times \Omega\right),
$$

where $u \in \mathscr{H}^{s+m-1, h}\left(I^{\prime} \times \Omega\right)$ and satisfies $(P-0)$.

Proof. Since

$$
A\left(t, x ; \phi^{m-1} u^{(k-1)} ; \partial\right)\left(u^{(k)}-u^{(k-1)}\right)=g_{k},
$$

we have from Proposition.1.4' and Lemma.2.4

$$
\begin{aligned}
& \left\|u^{(k)}-u^{(k-1)}\right\|_{\mathscr{H}^{s+m-2, h}(I / \times \Omega)}^{2} \\
& \quad \leqq C T^{\prime}\left\|u^{(k-1)}-u^{(k-2)}\right\|_{\mathscr{H}^{s+m-2, h}(I / \times \Omega)}^{2} .
\end{aligned}
$$

Taking $T^{\prime}$ small enough to satisfy $0<T^{\prime}<T_{1}$ and $C T^{\prime}<1,\left\{u^{(k)}\right\}$ is a Cauchy sequence in $\mathscr{H}^{s+m-2, h}\left(I^{\prime} \times \Omega\right)$. Moreover we have

$$
\|u\|_{\mathscr{H}^{s+m-1, h(I / \times \Omega)}} \leqq \underline{\lim }\left\|u^{(k)}\right\|_{\mathscr{H}^{s+m-1, h}\left(I^{\prime} \times \Omega\right)} \leqq M
$$

\section{§ 3. Local Existence Theorems for Quasi-linear Cauchy Problems with Degenerate-Structure-Generating Initial Data}

3.1. Condition $(k-*)$. Let us denote

$$
\mathscr{U}_{k}=\not_{t}^{m-1} \partial_{x}^{k} u \quad(k=0,1, \cdots), \quad \mathscr{U}=\left[\begin{array}{c}
\mathscr{U}_{0} \\
: \\
\mathscr{U}_{m-1}
\end{array}\right],
$$

where $\mathscr{U}_{k}$ is a $\mathscr{N}_{k}$-column vector and $\mathscr{U}$ is a $\mathscr{N}$-column vector, where $\mathscr{N}_{k}=m N_{k}$ 
$=m\left(\begin{array}{c}k+n-1 \\ k\end{array}\right)$ and $\mathscr{N}=\sum_{k=0}^{m-1} \mathscr{N}_{k}$. Then we have

Lemma 3.1. Let $p$ be a positive integer. Then

$$
\begin{aligned}
\partial_{x}^{p} & \{\mathscr{F}(t, x ; \mathscr{U}(t, x))\} \\
\quad & \left\{\Gamma_{p}\left(\mathscr{U}_{0}, \cdots, \mathscr{U}_{p+m-1} ; \partial_{x}, \partial_{\mathscr{U}}\right) \mathscr{F}\right\}(t, x ; \mathscr{U}(t, x)),
\end{aligned}
$$

where

$$
\begin{aligned}
& \Gamma_{p}=\sum_{q=0}^{p} \Gamma_{p q}\left(\mathscr{U}_{0}, \cdots, \mathscr{U}_{p+m-1} ; \partial_{x}, \partial_{\mathscr{U}}\right), \\
& \Gamma_{p q}=\sum_{r+s=q} \gamma_{p\left(r, k_{1}, \cdots, k_{s}\right)}\left(\mathscr{U}_{0}, \cdots, \mathscr{U}_{p+m-1}\right) \partial_{x}^{r} \partial_{\mathscr{U}_{k_{1}}} \cdots \partial_{\mathscr{U}_{k s}},
\end{aligned}
$$

where $\gamma_{p\left(r, k_{1}, \cdots, k_{s}\right)}$ is a $N_{p} \times\left(N_{r} \mathscr{N}_{k_{1}} \cdots \mathcal{N}_{k_{s}}\right)$-matrix with homogeneous polynomial entries of degree $s$ with respect to $\left\{\mathscr{U}_{0}, \cdots, \mathscr{U}_{p+m-1}\right\}$. Moreover, $\Gamma_{p}$ is represented as $\Gamma_{p}^{\prime}+\Gamma_{p}^{\prime \prime}$, where

$$
\begin{aligned}
& \Gamma_{p}^{\prime}=\sum_{q=0}^{p} \Gamma_{p q}^{\prime}, \quad \Gamma_{p}^{\prime \prime}=\sum_{q=0}^{p} \Gamma_{p q}^{\prime \prime}, \\
& \Gamma_{p q}^{\prime}=\sum_{\substack{r+s=q \\
\max _{\mathrm{k}_{i}<q}<q}} \gamma_{p\left(r, k_{1}, \ldots, k_{s}\right)}^{\prime}\left(\mathscr{U}_{0}, \cdots, \mathscr{U}_{p}\right) \partial_{x}^{r} \partial_{\mathscr{U}_{k_{1}}} \cdots \partial_{\mathscr{U}_{k s}}, \\
& \Gamma_{p q}^{\prime \prime}=\sum_{\substack{r+s=q \\
\max _{\mathfrak{k}} \geq q}} \gamma_{p\left(r, k_{1}, \ldots, k_{s}\right)}^{\prime \prime}\left(\mathscr{U}_{0}, \cdots, \mathscr{U}_{p+m-1}\right) \partial_{x}^{r} \partial_{\mathscr{U}_{k_{1}}} \cdots \partial_{\mathscr{U}_{k s}} .
\end{aligned}
$$

Proof. For simplicity, we consider the case when $n=1$, where $\mathscr{U}_{k}$ is a scalar valued function. By the differentiation of composite functions, we have

$$
\begin{aligned}
\partial_{x}^{p} & \{\mathscr{F}(t, x ; \mathscr{U}(t, x))\} \\
= & \left(\partial_{x}^{p} \mathscr{F}\right)(t, x ; \mathscr{U}(t, x))+\sum_{s=1}^{p} \sum_{\substack{r+\mu_{1}+\ldots+\mu_{s}=p \\
r \geqq 0, \mu_{l} \geqq 1}} \sum_{0 \leqq k_{1} \geqq m-1} c_{r \mu_{1} \cdots \mu_{3} k_{1} \cdots k_{s}} \\
& \times\left(\partial_{x}^{r} \partial_{\mathscr{U}_{k_{1}}} \cdots \partial_{\mathscr{U}_{k s}} \mathscr{F}\right)(t, x ; \mathscr{U}(t, x)) \mathscr{U}_{k_{1}+\mu_{1}} \cdots \mathscr{U}_{k_{s}+\mu_{s}},
\end{aligned}
$$

where $c_{r \mu_{1} \cdots \mu_{s} k_{1} \cdots k_{s}}$ is independent of $\{t, x, \mathscr{U}, \mathscr{F}\}$. We have only to remark that

$$
k_{i}+\mu_{i} \leqq \max k_{i}+p-r-(s-1) \leqq p \text { if } \max k_{i}<r+s .
$$

Let $k$ be a non-negative integer and let us say that $\mathcal{N}$ boundary functions $\mathscr{B}\left(t, x^{\prime}\right)$ satisfies $(k-*)$ with respect to $\mathscr{F}(t, x ; \mathscr{U})$ if

$$
\left(\partial_{x}^{r} \partial_{\mathscr{U}_{k_{1}}} \cdots \partial_{\mathscr{U}_{k s}} \mathscr{F}\right)\left(t, 0, x^{\prime} ; \mathscr{B}\left(t, x^{\prime}\right)\right)=0
$$


if $r+s \leqq \max \left(k-1, k_{1}, \ldots, k_{s}\right)$. Let us denote $\dot{\mathscr{U}}\left(t, x^{\prime}\right)=\mathscr{U}\left(t, 0, x^{\prime}\right)$. Then we have

Lemma 3.2. Assume that $\dot{\mathscr{U}}\left(t, x^{\prime}\right)$ satisfies $(k-*)$ with respect to $\mathscr{F}$. Then

$$
\begin{aligned}
\partial_{x}^{p} & \left.\{\mathscr{F}(t, x ; \mathscr{U}(t, x))\}\right|_{x_{1}=0} \\
\quad & =\sum_{q=k}^{p}\left\{\Gamma_{p q}^{\prime}\left(\dot{\mathscr{U}}_{0}\left(t, x^{\prime}\right), \cdots, \dot{\mathscr{U}}_{p}\left(t, x^{\prime}\right) ; \partial_{x}, \partial_{\mathscr{U}}\right) \mathscr{F}\right\}\left(t, 0, x^{\prime} ; \dot{\mathscr{U}}\left(t, x^{\prime}\right)\right) .
\end{aligned}
$$

Proof. Since

$$
\begin{aligned}
\partial_{x}^{p}\{\mathscr{F}(t, x ; \mathscr{U}(t, x))\} \\
=\sum_{q=0}^{p}\left\{\sum_{\substack{r+s=q \\
\max k_{l}<q}} \gamma_{p\left(r, k_{1}, \cdots, k_{s}\right)}^{\prime}\left(\mathscr{U}_{0}, \cdots, \mathscr{U}_{p}\right)\left(\partial_{x}^{r} \partial_{\mathscr{U}_{k_{1}}} \cdots \partial_{\mathscr{U}_{k s}} \mathscr{F}\right)(t, x ; \mathscr{U})\right. \\
\quad+\sum_{\substack{r+s=q \\
\max k_{l} \geqq q}} \gamma^{\prime \prime}{ }_{p\left(r, k_{1}, \cdots, k_{s}\right)}\left(\mathscr{U}_{0}, \cdots, \mathscr{U}_{p+m-1}\right)\left(\partial_{x}^{r} \partial_{\mathscr{U}_{k_{1}}} \cdots \partial_{\mathscr{U}_{k s}} \mathscr{F}\right)(t, x ; \mathscr{U}),
\end{aligned}
$$

the second part in the right hand side vanishes on $\left\{x_{1}=0\right\}$. Moreover, the summations of right hand side over $\{0 \leqq q \leqq k-1\}$ also vanish on $\left\{x_{1}=0\right\}$.

3.2. Fully degenerate condition (B). Now let us consider

$$
(P)\left\{\begin{array}{l}
A\left(t, x ; \phi^{m-1} u ; \partial\right) u=f\left(t, x ; \phi^{m-1} u\right) \quad \text { in } I \times \Omega, \\
\partial_{t}^{j} u=\phi_{j}(x)(j=0, \cdots, m-1) \text { on }\{t=0\} \times \Omega,
\end{array}\right.
$$

where we assume (A) stated in $\S 2$. Denoting

$$
\begin{aligned}
& \mathscr{A}(t, x ; \mathscr{U} ; \partial)=A(t, x ; U ; \partial), \quad \alpha_{j v}(t, x ; \mathscr{U})=a_{j v}(t, x ; U) \\
& \mathscr{F}(t, x ; \mathscr{U})=f(t, x ; U),
\end{aligned}
$$

let us say that $\mathscr{N}$ boundary functions $\mathscr{B}\left(t, x^{\prime}\right)$ satisfy (B)-fully degenerate condition -if

i) $\mathscr{B}$ satisfies $(|v|-*)$ with respect to $\alpha_{j v}$,

ii) $\mathscr{B}$ satisfies $(0-*)$ with respect to $\mathscr{F}$.

Let us denote

$$
\mathscr{U}_{p}(t, x)=\left(\partial_{x}^{p} u\right)(t, x), \quad \alpha_{j v, p}(t, x)=\left(\partial_{x}^{p} \alpha_{j v}\right)(t, x)
$$

where $\alpha_{j v}(t, x)=\alpha_{j v}(t, x ; \mathscr{U}(t, x))$. Then we have

$$
\begin{aligned}
\alpha_{j v, p}(t, x) & =\left\{\Gamma_{p}\left(\mathscr{U}_{0}, \cdots, \mathscr{U}_{p+m-1} ; \partial_{x}, \partial_{\mathscr{U}}\right) \alpha_{j v}\right\}(t, x ; \mathscr{U}) \\
& =\sum_{k=0}^{p}\left\{\Gamma_{p k}\left(\mathscr{U}_{0}, \cdots, \mathscr{U}_{p+m-1} ; \partial_{x}, \partial_{\mathscr{U}}\right) \alpha_{j v}\right\}(t, x ; \mathscr{U})
\end{aligned}
$$

Let us consider 


$$
\Gamma_{r}=\left(\Gamma_{r}\right)_{|r|=r},
$$

where $r=\left(r_{1}, \ldots, r_{n}\right)$, then we have

$$
\begin{aligned}
\partial_{x}^{r} & \left\{\mathscr{A}(t, x ; \mathscr{U}(t, x) ; \partial) u(t, x)-\partial_{t}^{m} u(t, x)\right\} \\
& =\partial_{x}^{r}\left\{\sum_{\substack{j+|v| \leqq m \\
j \leqq m}} \alpha_{j v}(t, x ; \mathscr{U}(t, x)) \partial_{t}^{j} \partial_{x}^{v} u(t, x)\right\} \\
& =\sum_{\substack{j+|v|=m \\
j \leqq m-1}} \sum_{p+q=r} \Gamma !(p ! q !)^{-1} \partial_{t}^{j} \partial_{x}^{v+q} u(t, x) \alpha_{j v, p}(t, x) \\
& =\sum_{\substack{j+|v|=m \\
j \leqq m-1}}\left\{\sum_{p+q=r} \Gamma !(p ! q !)^{-1} \partial_{t}^{j} \partial_{x}^{v+q} u(t, x)\right. \\
& \left.=\sum_{\substack{j+|v|=m \\
j \leqq m-1}} \Gamma_{r(j, v)}\left(\mathscr{U}_{0}, \cdots, \mathscr{U}_{|r|+m} ; \partial_{x}, \partial_{\mathscr{U}}\right)\right\} \alpha_{j v}\left(t, x ; \mathscr{U}_{p}\right) .
\end{aligned}
$$

Let us denote

$$
\begin{aligned}
& \Gamma_{r(j, v)}=\left(\Gamma_{r(j, v)}\right)_{|r|=r}, \\
& \Gamma_{r(j, v)}^{\prime}=\left(\Gamma_{r(j, v)}^{\prime}\right)_{|r|=r}, \Gamma_{r(j, v)}^{\prime \prime}=\left(\Gamma_{r(j, v)}^{\prime \prime}\right)_{|r|=r}
\end{aligned}
$$

for $j+|v| \leqq m$, where

$$
\begin{aligned}
\Gamma_{r(j, v)} & =\Gamma_{r(j, v)}\left(\mathscr{U}_{0}, \cdots, \mathscr{U}_{r+m} ; \partial_{x}, \partial_{\mathscr{U}}\right) \\
& =\sum_{p+q=r} r !(p ! q !)^{-1} \partial_{t}^{j} \partial_{x}^{v+q} u \Gamma_{p}\left(\mathscr{U}_{0}, \cdots, \mathscr{U}_{|p|+m-1} ; \partial_{x}, \partial_{\mathscr{U}}\right), \\
\Gamma_{r(j, v)}^{\prime} & =\Gamma_{r(j, v)}^{\prime}\left(\mathscr{U}_{0}, \cdots, \mathscr{U}_{r+m} ; \partial_{x}, \partial_{U_{U}}\right) \\
& =\sum_{p+q=r} r !(p ! q !)^{-1} \partial_{t}^{j} \partial_{x}^{v+q} u \Gamma_{p}^{\prime}\left(\mathscr{U}_{0}, \cdots, \mathscr{U}_{|p|} ; \partial_{x}, \partial_{\mathscr{U}}\right), \\
\Gamma_{r^{\prime(j, v)}}^{\prime \prime} & =\Gamma_{r(j, v)}^{\prime \prime}\left(\mathscr{U}_{0}, \cdots, \mathscr{U}_{r+m} ; \partial_{x}, \partial_{\mathscr{U}}\right) \\
& =\sum_{p+q=r} r !(p ! q !)^{-1} \partial_{t}^{j} \partial_{x}^{v+q} u \Gamma_{p}^{\prime \prime}\left(\mathscr{U}_{0}, \cdots, \mathscr{U}_{|p|+m-1} ; \partial_{x}, \partial_{\mathscr{U}}\right) .
\end{aligned}
$$

Moreover, we denote

$$
\Gamma_{r(j, v)}^{ \pm \pm}=\left(\Gamma_{r(j, v)}^{\prime}\right)_{|r|=r}
$$

where 


$$
\begin{aligned}
& \Gamma_{r^{(j, v)}}^{\prime+}=\Gamma_{r(j, v)}^{\prime+}\left(\mathscr{U}_{0}, \cdots, \mathscr{U}_{r} ; \partial_{x}, \partial_{\mathscr{U}}\right) \\
& \quad=\sum_{p+q=r} r !(p ! q !)^{-1} \partial_{t}^{j} \partial_{x}^{v+q} u \sum_{k=|v|}^{|p|} \Gamma_{p_{k}}^{\prime}\left(\mathscr{U}_{0}, \cdots, \mathscr{U}_{|p|} ; \partial_{x}, \partial_{\mathscr{U}}\right), \\
& \Gamma_{\left.r^{\prime}, v, v\right)}^{\prime-}=\Gamma_{r(j, v)}^{\prime-}\left(\mathscr{U}_{0}, \cdots, \mathscr{U}_{r+m} ; \partial_{x}, \partial_{\mathscr{U}}\right) \\
& \quad=\sum_{p+q=r} r !(p ! q !)^{-1} \partial_{t}^{j} \partial_{x}^{v+q} u \sum_{k=0}^{|v|-1} \Gamma_{p_{k}}^{\prime}\left(\mathscr{U}_{0}, \cdots, \mathscr{U}_{|p|} ; \partial_{x}, \partial_{U_{U}}\right),
\end{aligned}
$$

Let us denote

$$
\begin{aligned}
\beta_{r}\left(\mathscr{U}_{0}, \cdots, \mathscr{U}_{r+m} ; t, x ; \mathscr{U}\right) \\
\quad=\sum_{\substack{j+|v|=m \\
j \leqq m-1}}\left\{\Gamma_{r(j, v)}\left(\mathscr{U}_{0}, \cdots, \mathscr{U}_{r+m} ; \partial_{x}, \partial_{\mathscr{V}}\right) \alpha_{j v}\right\}(t, x ; \mathscr{V})
\end{aligned}
$$

and $\beta_{r}^{\prime}, \beta_{r}^{\prime \prime}, \beta_{r}^{\prime \pm}$ are defined analogously, then we have

Lemma 3.3. Let us assume that $\mathscr{B}\left(t, x^{\prime}\right)$ satisfies (B). Then

i) $\beta_{r}\left(\mathscr{U}_{0}, \cdots, \mathscr{U}_{r+m} ; t, 0, x^{\prime} ; \mathscr{B}\left(t, x^{\prime}\right)\right)$

$=\beta_{r}^{\prime+}\left(\mathscr{U}_{0}, \cdots, \mathscr{U}_{r} ; t, 0, x^{\prime} ; \mathscr{B}\left(t, x^{\prime}\right)\right)$.

ii) $\quad\left\{\Gamma_{r}\left(\mathscr{U}_{0}, \cdots, \mathscr{U}_{r+m-1} ; \partial_{x}, \partial_{\mathscr{U}}\right) \mathscr{F}\right\}\left(t, 0, x^{\prime} ; \mathscr{B}\left(t, x^{\prime}\right)\right)$

$=\left\{\Gamma_{r}^{\prime}\left(\mathscr{U}_{0}, \cdots, \mathscr{U}_{r} ; \partial_{x}, \partial_{\mathscr{U}}\right) \mathscr{F}\right\}\left(t, 0, x^{\prime} ; \mathscr{B}\left(t, x^{\prime}\right)\right)$.

Let us denote

$$
\begin{aligned}
& \mathscr{F}_{r}\left(\mathscr{U}_{0}, \cdots, \mathscr{U}_{r} ; t, x ; \mathscr{V}\right) \\
&=-\beta_{r}^{+}\left(\mathscr{U}_{0}, \cdots, \mathscr{U}_{r} ; t, x ; \mathscr{V}\right)+\left\{\Gamma_{r}^{\prime}\left(\mathscr{U}_{0}, \cdots, \mathscr{U}_{r} ; \partial_{x}, \partial_{\mathscr{V}}\right) \mathscr{F}\right\}(t, x ; \mathscr{V}), \\
& \mathscr{H}_{r}\left(\mathscr{U}_{0}, \cdots, \mathscr{U}_{r+m} ; t, x ; \mathscr{V}\right) \\
&=-\beta_{r}^{\prime-}\left(\mathscr{U}_{0}, \cdots, \mathscr{V}_{r+m} ; t, x ; \mathscr{V}\right)-\beta_{r}^{\prime \prime}\left(\mathscr{U}_{0}, \cdots, \mathscr{U}_{r+m} ; t, x ; \mathscr{V}\right), \\
& \quad+\left\{\Gamma_{r}^{\prime}\left(\mathscr{U}_{0}, \cdots, \mathscr{U}_{r} ; \partial_{x}, \partial_{\mathscr{V}}\right) \mathscr{F}\right\}(t, x ; \mathscr{V}),
\end{aligned}
$$

then we have

Corollary. Let us assume $\mathscr{B}\left(t, x^{\prime}\right)$ satisfies $(B)$, then

$$
\mathscr{H}_{r}\left(\mathscr{U}_{0}, \cdots, \mathscr{U}_{r+m} ; t, 0, x^{\prime} ; \mathscr{B}\left(t, x^{\prime}\right)\right)=0 .
$$

Hence we have

Proposition 3.4. Assume that $u(t, x)$ is a solution of $(P)$ and that

$$
\dot{\mathscr{U}}\left(t, x^{\prime}\right)=\phi_{t}^{m-1} \phi_{x}^{m-1} u\left(t, 0, x^{\prime}\right)
$$

satisfies $(B)$, then $\left\{\dot{u}_{j}\left(t, x^{\prime}\right)=\left(\partial_{x}^{j} u\right)\left(t, 0, x^{\prime}\right)(j=0,1,2, \cdots)\right\}$ satisfy $(Q)$ : 
$(Q)\left\{\begin{array}{l}\partial_{t}^{m} \dot{u}_{r}=\mathscr{F}_{r}\left(\phi_{t}^{m-1} \dot{u}_{0}, \ldots, \phi_{t}^{m-1} \dot{u}_{r} ; t, 0, x^{\prime} ; \phi_{t}^{m-1} \dot{u}_{0}, \ldots, \phi_{t}^{m-1} \dot{u}_{m-1}\right) \text { in } I \times R^{n-1}, \\ \partial_{t}^{j} \dot{u}_{r}=\dot{\phi}_{j, r}\left(x^{\prime}\right) \quad(j=0, \ldots, m-1) \quad \text { on }\{t=0\} \times R^{n-1} \quad(r=0,1,2, \ldots),\end{array}\right.$ where

$$
\dot{\phi}_{j, r}\left(x^{\prime}\right)=\left(\partial_{x}^{r} \phi_{j}\right)\left(0, x^{\prime}\right)
$$

Let us denote

$\left(Q_{h}\right)\left\{\begin{array}{l}\partial_{t}^{m} \dot{u}_{r}=\mathscr{F}_{r}\left(\phi_{t}^{m-1} \dot{u}_{0}, \cdots, \phi_{t}^{m-1} \dot{u}_{r} ; t, 0, x^{\prime} ; \phi_{t}^{m-1} \dot{u}_{0}, \cdots, \phi_{t}^{m-1} \dot{u}_{m-1}\right) \text { in } I \times R^{n-1}, \\ \partial_{t}^{j} \dot{u}_{r}=\dot{\phi}_{j, r}\left(x^{\prime}\right)(j=0, \cdots, m-1) \text { on }\{t=0\} \times R^{n-1}(r=0,1,2, \cdots, h-1) .\end{array}\right.$

Then $\left(Q_{h}\right)$ is a Cauchy problem for a system of ordinary differential equations with respect to $t$ with unknown functions $\left\{\dot{u}_{r}(r=0,1, \cdots, h-1)\right\}$. Therefore, it is uniquely solvable in locale for any $h(\geqq m)$.

\section{3. $\sigma$-structure-generating initial data.}

Proposition 3.5. Let $\left\{g_{j}\left(t, x^{\prime}\right)(j=0,1, \cdots)\right\}$ be a solution of $(Q)$. Assume that $g\left(t, x^{\prime}\right)=\phi_{t}^{m-1}\left(g_{0}\left(t, x^{\prime}\right), \cdots, g_{m-1}\left(t, x^{\prime}\right)\right)$ satisfies $(B)$. Let $u$ be a solution of $(P)$, then we have

$$
\dot{u}_{j}=g_{j} \quad(j=0,1,2, \ldots) .
$$

Proof. Since $u$ satisfies $(P)$, we have

$$
\begin{aligned}
\partial_{t}^{m} \dot{u}_{r}= & \mathscr{F}_{r}\left(\phi_{t}^{m-1} \dot{u}_{0}, \cdots, \phi_{t}^{m-1} \dot{u}_{r} ; t, 0, x^{\prime} ; \dot{\mathscr{U}}\right) \\
& +\mathscr{H}_{r}\left(\phi_{t}^{m-1} \dot{u}_{0}, \cdots, \phi_{t}^{m-1} \dot{u}_{r+m} ; t, 0, x^{\prime} ; \mathscr{U}\right) .
\end{aligned}
$$

On the other hand, since $g$ satisfies $(B)$, we have

$$
\mathscr{H}_{r}\left(\not_{t}^{m-1} \dot{u}_{0}, \cdots, \phi_{t}^{m-1} \dot{u}_{r+m} ; t, 0, x^{\prime} ; g\right)=0,
$$

therefore,

$$
\begin{aligned}
\partial_{t}^{m} g_{r} & =\mathscr{F}_{r}\left(\phi_{t}^{m-1} g_{0}, \cdots, \phi_{t}^{m-1} g_{r} ; t, 0, x^{\prime} ; g\right) \\
& +\mathscr{H}_{r}\left(\phi_{t}^{m-1} \dot{u}_{0}, \cdots, \phi_{t}^{m-1} \dot{u}_{r+m} ; t, 0, x^{\prime} ; g\right) .
\end{aligned}
$$

Hence we have

$$
\begin{aligned}
& \partial_{t}^{m}\left(\dot{u}_{r}-g_{r}\right) \\
&=\left\{\mathscr{F}_{r}\left(\phi_{t}^{m-1} \dot{u}_{0}, \cdots, \phi_{t}^{m-1} \dot{u}_{r} ; t, 0, x^{\prime} ; \dot{\mathscr{U}}\right)\right. \\
&\left.-\mathscr{F}_{r}\left(\phi_{t}^{m-1} g_{0}, \cdots, \phi_{t}^{m-1} g_{r} ; t, 0, x^{\prime} ; g\right)\right\} \\
&+\left\{\mathscr{H}_{r}\left(\phi_{t}^{m-1} \dot{u}_{0}, \cdots, \phi_{t}^{m-1} \dot{u}_{r+m} ; t, 0, x^{\prime} ; \mathscr{U}\right)\right. \\
&\left.-\mathscr{H}_{r}\left(\phi_{t}^{m-1} \dot{u}_{0}, \cdots, \phi_{t}^{m-1} \dot{u}_{r+m} ; t, 0, x^{\prime} ; g\right)\right\} .
\end{aligned}
$$


Let us define a system of ordinary differential equations with respect to $v$ $=\left(v_{0}, \cdots, v_{m-1}\right)$ :

$$
\left(\widetilde{Q}_{m}\right)\left\{\begin{array}{c}
\partial_{t}^{m} v_{r}=\left\{\mathscr{F}_{r}\left(\phi_{t}^{m-1}\left(v_{0}+g_{0}\right), \cdots, \phi_{t}^{m-1}\left(v_{r}+g_{r}\right) ; t, 0, x^{\prime} ; \phi_{t}^{m-1} v+g\right)\right) \\
\left.-\mathscr{F}_{r}\left(\phi_{t}^{m-1} g_{0}, \cdots, \phi_{t}^{m-1} g_{r} ; t, 0, x^{\prime} ; g\right)\right\} \\
+\left\{\mathscr{H}_{r}\left(\phi_{t}^{m-1} \dot{u}_{0}, \cdots, \phi_{t}^{m-1} \dot{u}_{r+m} ; t, 0, x^{\prime} ; \phi_{t}^{m-1} v+g\right)\right. \\
\left.-\mathscr{H}_{r}\left(\phi_{t}^{m-1} \dot{u}_{0}, \cdots, \phi_{t}^{m-1} \dot{u}_{r+m} ; t, 0, x^{\prime} ; g\right)\right\} \\
\left(\phi_{t}^{m-1} v_{r}\right)\left(0, x^{\prime}\right)=0 \quad(r=0, \cdots, m-1),
\end{array}\right.
$$

where $\left\{\dot{u}_{j}, g_{j}\right\}$ are considered as given functions, then

$$
v=\left(\dot{u}_{0}-g_{0}, \cdots, \dot{u}_{m-1}-g_{m-1}\right)
$$

satisfies $\left(\widetilde{Q}_{m}\right)$. On the other hand, it is obvious that $v=0$ is the unique solution of $\left(\widetilde{Q}_{m}\right)$. Hence we have $\left\{\dot{u}_{r}=g_{r}\right\}_{r=0,1, \ldots, m-1}$. The fact that $\left\{\dot{u}_{r}=g_{r}\right\}_{r \geqq m}$ follows from Proposition.3.4.

Set

$$
g^{(h)}(t, x)=\sum_{r=0}^{h-1}(r !)^{-1} \tilde{\rho}\left(x_{1}\right)^{r} g_{(r, 0, \cdots, 0)}\left(t, x^{\prime}\right)
$$

and

$$
G^{(h)}(t, x)=\not^{m-1} g^{(h)}(t, x), g^{(h)}(t, x)=\phi_{t}^{m-1} \phi_{x}^{m-1} g^{(h)}(t, x),
$$

where $\left\{g_{r}=\left(g_{r}\right)_{|r|=r}(r=0,1, \cdots, h-1)\right\}$, is a solution of $\left(Q_{h}\right)$, then we have $g^{(h)}\left(t, 0, x^{\prime}\right)=g\left(t, x^{\prime}\right)$. Let us assume that $g$ satisfies $(B)$, then $(P)$ is reduced to $(\widetilde{P})_{g^{(h)}}\left\{\begin{array}{l}\tilde{A}\left(t, x ; \phi^{m-1} v ; \partial\right) v=\tilde{f}\left(t, x ; \phi^{m-1} v\right) \quad \text { in } I \times \Omega, \\ \partial_{t}^{j} v=\widetilde{\phi}_{j}(x)(j=0, \cdots, m-1) \quad \text { on } \cdot\{t=0\} \times \Omega,\end{array}\right.$

where

$$
\begin{aligned}
& \tilde{A}(t, x ; V ; \partial)=A\left(t, x ; G^{(h)}(t, x)+V ; \partial\right), \\
& \tilde{f}(t, x ; V)=-A\left(t, x ; G^{(h)}(t, x)+V ; \partial\right) g^{(h)}+f\left(t, x ; G^{(h)}(t, x)+V\right), \\
& \tilde{\phi}_{j}(x)=\phi_{j}(x)-\left(\partial_{t}^{j} g^{(h)}\right)(0, x) .
\end{aligned}
$$

Moreover, from the definition of $\left(Q_{h}\right)$, we have

Lemma 3.6. Let $\left\{g_{r}(r=0,1, \cdots, h-1)\right\}$ be a solution of $\left(Q_{h}\right)$, where $g$ satisfies $(B)$, then $(\widetilde{P})_{g^{(h)}}$ satisfies $(h-D)($ defined in $\S 2)$.

Let us say that initial data $\left\{\phi_{j}\right\}_{j=0,1, \cdots, m-1}$ satisfy $(\sigma-S-G)-\sigma$-structuregenerating condition -, if

i) $g=\phi_{t}^{m-1}\left(g_{0}, \cdots, g_{m-1}\right)$ satisfies $(B)$, where $\left\{g_{r}(r=0,1, \cdots, m-1)\right\}$ is a solution 
of $\left(Q_{m}\right)$,

ii) $(\widetilde{P})_{g^{(h)}}$ satisfies $(\sigma-S)$ (defined in $\left.\S 2\right)$.

Here we have from Theorem 1

Theorem 2. Let us assume $(A)$ and assume that $\left\{\phi_{j}\right\}_{j=0,1, \cdots, m-1}$ satisfy $(\sigma$ $-S-G)$, then there exists an interval $I_{s}=\left(0, T_{s}\right)$ for any $s\left(\geqq s_{0}\right)$ such that there exists a unique solution of $(P)$ in $H^{s}\left(I_{s} \times \Omega\right)$. Moreover the boundary functions $\left\{\dot{u}_{r}=\partial_{x}^{r} u\left(t, 0, x^{\prime}\right)\right\}$ of the solution $u$ satisfy $(Q)$.

\section{\$4. Blow-up of Solutions}

Let us consider

$$
\left\{\begin{array}{l}
\partial_{t}^{2} u-a\left(u, u_{x}, u_{y}\right)^{2}\left\{\partial_{x}^{2}+\partial_{y}^{2}\right\} u=f\left(u, u_{x}, u_{y}\right) \quad \text { in }\{t>0, x>0, y \in R\}, \\
\partial_{t}^{j} u=\phi_{j}(x, y) \quad(j=0,1) \quad \text { on }\{t=0, x>0, y \in R\},
\end{array}\right.
$$

where $a(u, v, w)$ and $f(u, v, w)$ are homogeneous polynomials of degree $p(\geqq 1)$ and $q(\geqq 2)$, then the fully degenerate condition is

$$
\left\{\begin{array}{c}
a(u, v, w)=0 \\
f_{v}(u, v, w)=0, \\
f_{w}(u, v, w)=0
\end{array}\right.
$$

and $\left(Q_{1}\right)$ is

$$
\left\{\begin{aligned}
\partial_{t}^{2} u & =f(u, v, w) \\
\partial_{t}^{2} v & =f_{u}(u, v, w) v \\
\partial_{t}^{2} w & =f_{u}(u, v, w) w
\end{aligned}\right.
$$

in $\{0<t<T, y \in R\}$ with initial conditions on $\{t=0, y \in R\}$ :

$$
\partial_{t}^{j} u=\phi_{j}(0, y), \partial_{t}^{j} v=\phi_{j x}(0, y), \partial_{t}^{j} w=\phi_{j y}(0, y) \quad(j=0,1) .
$$

Let us define

$(B-1):\{u \neq 0, v=\alpha u, w=\beta u\}$, where $\alpha$ and $\beta$ are constants satisfying

$$
\left\{a(1, \alpha, \beta)=0, f_{v}(1, \alpha, \beta)=0, f_{w}(1, \alpha, \beta)=0\right\},
$$

$(B-2):\{u=0, v \neq 0, w=\gamma v\}$, where $\gamma$ is a constant satisfying

$$
\left\{a(0,1, \gamma)=0, f_{v}(0,1, \gamma)=0, f_{w}(0,1, \gamma)=0\right\} .
$$

Then $(B)$ is satisfied if $(B-1)$ or $(B-2)$ is satisfied.

Let a solution of $\left(Q_{1}\right)$ satisfy $(B-1)$, then (\#) is reduced to 


$$
\left\{\begin{array}{c}
\partial_{t}^{2} u-q^{-1} f_{u}(1, \alpha, \beta) u^{q}=0 \\
\alpha\left\{\partial_{t}^{2} u-f_{u}(1, \alpha, \beta) u^{q}\right\}=0 \\
\beta\left\{\partial_{t}^{2} u-f_{u}(1, \alpha, \beta) u^{q}\right\}=0
\end{array}\right.
$$

because $q f(1, \alpha, \beta)=f_{u}(1, \alpha, \beta)$. Therefore we have a contradiction, if $f_{u}(1, \alpha, \beta) \neq 0,(\alpha, \beta) \neq(0,0)$ and $u \neq 0$. If $\alpha=\beta=0$, (\#) is reduced to

$$
\partial_{t}^{2} u=q^{-1} f_{u}(1,0,0) u^{q} .
$$

Let a solution of $\left(Q_{1}\right)$ satisfy $(B-2)$. Then $(\#)$ is reduced to

$$
\partial_{t}^{2} v=f_{u}(0,1, \gamma) v^{q}
$$

Hereafter, we consider $(P)$ on the assumption

$$
(A-1): a(1,0,0)=f_{v}(1,0,0)=f_{w}(1,0,0)=0, f_{u}(1,0,0)>0,
$$

or

$$
(A-2): a(0,1,0)=f_{v}(0,1,0)=f_{w}(0,1,0)=0, f_{u}(0,1,0)>0 .
$$

Let us consider

$$
\left\{\begin{array}{l}
\tau^{\prime \prime}=\lambda \tau^{q} \quad \text { in }\{t>0\} \\
\tau=\alpha, \tau^{\prime}=\beta \quad \text { at } t=0
\end{array}\right.
$$

where $\lambda$ is a positive constant. $(\tau)$ is solvable by direct integration:

$$
t= \pm \int_{\alpha}^{\tau} Q(\tau)^{-1 / 2} d \tau
$$

where

$$
Q(\tau)=2 \lambda /(q+1) \tau^{q+1}+\beta^{2}-2 \lambda /(q+1) \alpha^{q+1} .
$$

First, we consider the case when $q$ is odd. Then let us define

$$
\alpha(\beta)= \begin{cases}-\left\{(q+1) /(2 \lambda) \beta^{2}\right\}^{1 /(q+1)} & \text { if } \beta \geqq 0, \\ \left\{(q+1) /(2 \lambda) \beta^{2}\right\}^{1 /(q+1)} & \text { if } \beta<0,\end{cases}
$$

then the above solution blows up in finite time if $\alpha \neq \alpha(\beta)$. More precisely, let us define

$$
t^{*}=t^{*}(\alpha, \beta)= \begin{cases}\int_{\alpha}^{\infty} Q(\tau)^{-1 / 2} d \tau \quad \text { if } \beta \geqq 0, \\ \int_{\tau(\alpha, \beta)}^{\alpha} Q(\tau)^{-1 / 2} d \tau+\int_{\tau(\alpha, \beta)}^{\infty} Q(\tau)^{-1 / 2} d \tau \quad \text { if } \beta<0,\end{cases}
$$


when $\alpha>\alpha(\beta)$, and $t^{*}(-\alpha,-\beta)=t^{*}(\alpha, \beta)$ when $\alpha<\alpha(\beta)$, where $\tau(\alpha, \beta)$ is a positive root of $Q(\tau)=0$. Then we have

Lemma 4.1. Let $q$ be odd.

i) In case when $\beta>\beta(\alpha)$, there exists a solution of $(\tau)$ in $\left(0, t^{*}\right)$ and $\tau(t) \rightarrow+\infty$ as $t \rightarrow t^{*}$

ii) In case when $\beta<\beta(\alpha)$, there exists a solution of $(\tau)$ in $\left(0, \mathrm{t}^{*}\right)$ and $\tau(t) \rightarrow-\infty$ as $t \rightarrow t^{*}$.

Next, we consider the case when $q$ is even. Let us define

$$
\alpha(\beta)=\left\{(q+1) /(2 \lambda) \beta^{2}\right\}^{1 /(q+1)} .
$$

Then the above solution blows up in finite time if $\alpha \neq \alpha(\beta)$. More precisely, let us define

$$
t^{*}=t^{*}(\alpha, \beta)= \begin{cases}\int_{\alpha}^{\infty} Q(\tau)^{-1 / 2} d \tau \quad \text { if } \beta \geqq 0, \\ \int_{\tau(\alpha, \beta)}^{\alpha} Q(\tau)^{-1 / 2} d \tau+\int_{\tau(\alpha, \beta)}^{\infty} Q(\tau)^{-1 / 2} d \tau & \text { if } \beta<0,\end{cases}
$$

where $\tau(\alpha, \beta)$ is a real root of $Q(\tau)=0$.

Lemma 4.2. Let $q$ be even and let $\alpha \neq \alpha(\beta)$, then there exists a solution of $(\tau)$ in $\left(0, t^{*}\right)$ such that

$$
\tau(t) \rightarrow+\infty \quad \text { as } t \rightarrow t^{*}
$$

Hence we have

\section{Theorem 3.}

i) Set $\lambda=q^{-1} f_{u}(1,0,0)$ under the assumption (A.1). Let us assume that

$$
\phi_{0}(0, y) \neq \alpha\left(\phi_{1}(0, y)\right) .
$$

Then there exists no $C^{2}((0, \infty) \times \bar{\Omega})$-solution of $(P)$. More precisely, let $y_{0}$ satisfy $\left(C_{y_{0}}\right)$

$$
\phi_{0}\left(0, y_{0}\right) \neq \alpha\left(\phi_{1}\left(0, y_{0}\right)\right) \text {. }
$$

Then

$$
\left|u\left(t, 0, y_{0}\right)\right| \rightarrow \infty \quad \text { as } t \rightarrow t^{*}\left(\phi_{0}\left(0, y_{0}\right), \phi_{1}\left(0, y_{0}\right)\right) .
$$

ii) Set $\lambda=f_{u}(0,1,0)$ under the assumption (A. 2). Let us assume that

$$
\phi_{0 x}(0, y) \neq \alpha\left(\phi_{1 x}(0, y)\right) \text {. }
$$

Then there exists no $C^{2}((0, \infty) \times \bar{\Omega})$-solution of $(P)$. More precisely, let $y_{0}$ satisfy 
$\left(C_{y_{0}}^{\prime}\right)$

$$
\phi_{0 x}\left(0, y_{0}\right) \neq \alpha\left(\phi_{1 x}\left(0, y_{0}\right)\right) .
$$

Then

$$
\left|u_{x}\left(t, 0, y_{0}\right)\right| \rightarrow \infty \quad \text { as } t \rightarrow t^{*}\left(\phi_{0 x}\left(0, y_{0}\right), \phi_{1 x}\left(0, y_{0}\right)\right) .
$$

In the following let us consider an example:

where

$$
\left\{\begin{array}{l}
u_{t t}-u^{2} u_{x x}=\kappa u u_{x}^{2}(\kappa>0) \quad \text { in }\{t>0,0<x<1\}, \\
u(0, x)=\phi_{0}(x), u_{t}(0, x)=\phi_{1}(x)
\end{array}\right.
$$

$$
\begin{aligned}
& \phi_{0}(0)=\phi_{0}(1)=0, \phi_{0}^{\prime}(0) \neq 0, \phi_{0}^{\prime}(1) \neq 0, \phi_{0}(x) \neq 0,(0<x<1), \\
& \phi_{1}(0)=\phi_{1}(1)=0 .
\end{aligned}
$$

Then we have

$(B):\{u(t, 0)=u(t, 1)=0\}$,

$$
\left\{\begin{array}{l}
u_{t t}(t, 0)=0, u_{x t t}(t, 0)=\kappa u_{x}(t, 0)^{3}, \\
u_{t t}(t, 1)=0, u_{x t t}(t, 1)=\kappa u_{x}(t, 1)^{3},
\end{array}\right.
$$

and $\left\{\phi_{0}, \phi_{1}\right\}$ satisfies $(1-S-G)$. Hence there exists a smooth local solution, which blows-up in finite time.

We remark that this equation has a periodical solution in time, belonging to $C^{0}(R \times[0,1]) \cap C^{\infty}(R \times(0,1))$ :

Proposition 4.3. Let T be any positive number. Then there exists a solution $u$ of the equation:

$$
u_{t t}-u^{2} u_{x x}=\kappa u u_{x}^{2}(\kappa>0) \quad \text { in }\{t \in R, 0<x<1\},
$$

satisfying

$$
u(t+T, x)=u(t, x), u(t, 1-x)=u(t, x), u(t, 0)=u(t, 1)=0 .
$$

Proof. Set $u(t, x)=\tau(t) \xi(x)$ and

$$
\frac{\tau^{\prime \prime}}{\tau^{3}}=\xi \xi^{\prime \prime}+\kappa \xi^{\prime 2}=-\sigma,
$$

where $\sigma$ is an arbitrary positive constant.

First we integrate $\tau^{\prime \prime}=-\sigma \tau^{3}$, and then we have

$$
t= \pm \int_{0}^{\tau}\left\{\sigma / 2\left(a^{4}-\tau^{4}\right)\right\}^{-1 / 2} d \tau,
$$

where $a$ is an arbitrary positive constant. It defines a periodic function $\tau(t) \in C^{\infty}(R)$ with period 


$$
T=4 a^{-1}(\sigma / 2)^{-1 / 2} \int_{0}^{1}\left(1-\tau^{4}\right)^{-1 / 2} d \tau .
$$

Next, we consider $\xi \xi^{\prime \prime}+\kappa \xi^{\prime 2}=-\sigma$. Then we have

$$
\xi^{\prime \prime} / \xi^{\prime}+\kappa \xi^{\prime} / \xi=-\sigma /\left(\xi \xi^{\prime}\right),
$$

and hence,

$$
\left(\log \left|\xi^{\kappa} \xi^{\prime}\right|\right)^{\prime}=-\sigma\left(\xi^{2 \kappa-1} \xi^{\prime}\right)\left(\xi^{\kappa} \xi^{\prime}\right)^{-2}
$$

that is,

$$
\eta^{\prime}=-\sigma(2 \kappa)^{-1}\left(\xi^{2 \kappa}\right)^{\prime} e^{-2 \eta}
$$

where $\eta=\log \left|\xi^{\kappa} \xi^{\prime}\right|$. Therefore integrating

$$
\left(e^{2 \eta} / 2\right)^{\prime}=-\sigma(2 \kappa)^{-1}\left(\xi^{2 \kappa}\right)^{\prime},
$$

we have

$$
e^{2 \eta}=(\sigma / \kappa)\left(b^{2 \kappa}-\xi^{2 \kappa}\right),
$$

where $b$ is an arbitrary positive constant, that is,

$$
\left(\xi^{\kappa} \xi^{\prime}\right)^{2}=(\sigma / \kappa)\left(b^{2 \kappa}-\xi^{2 \kappa}\right) .
$$

Hence we have

$$
x= \pm \int_{0}^{\xi} \xi^{\kappa}\left\{(\sigma / \kappa)\left(b^{2 \kappa}-\xi^{2 \kappa}\right)\right\}^{-1 / 2} d \xi
$$

where

$$
b=(\sigma / \kappa)^{1 / 2} / 2\left\{\int_{0}^{1} \xi^{\kappa}\left(1-\xi^{2 \kappa}\right) d \xi\right\}^{-1} .
$$

Moreover, we have $\xi(x) \in C^{\infty}(0,1)$ and

$$
\xi(x)^{\kappa+1} /\{x(1-x)\} \rightarrow(\kappa+1)\left(\sigma / \kappa b^{2 \kappa}\right)^{1 / 2}
$$

as $x \rightarrow 0$ or $x \rightarrow 1$.

\section{References}

[1] Ebihara, Y., Local classical solutions to degenerate quasilinear wave equations, Hukudairishuho, 16 (1986), 1-15.

[2] Nonaka, Y., Local solutions for degenerate quasilinear wave equations, to appear.

[3] Sakamoto, R., Hyperbolic Cauchy problems in a region with characteristic boundary of full multicity, Math. J. Kyoto Univ., 29 (1989).

[4] Makino, T., On a local existence theorem for the evolution equation of gaseous stars, Pattern and waves, Kinokuniya/North-Holland (1986), 459-479. 
[5] Makino, T., Ukai S., et Kawashima, S., Sur la solution à support compact de l'equation d'Euler compressible, Japan J. Appl. Math., 3 (1986), 249-257.

[6] Craig, W., Nonstrictly hyperbolic nonlinear systems, Math. Ann. 277 (1987), 213-232. 
